\title{
Elementos sobre epistemología y enseñanza de la educación física.
}

\author{
Elements about epistemology and teaching of physical education
}

Victor Jairo Chinchilla G."

\section{Resumen}

En la discusión sobre hechos que transforman la educación fisica en la Universidad Pedagógica Nacional y en el contexto nacional, el tema de la relación entre epistemologia y educación física es de importancia fundamental. Este artículo presenta los conceptos de la epistemología científica a partir de los cuales se ha desarrollado la discusión de la educación física y su objeto de estudio, y plantea la opción de considerar la educación fisica como una práctica. Para ello se apoya en la caracterización que hace Pierre Parlebas y en el concepto de episteme según Michel Foucault. Plantea una metodologia de investigación desde los campos de la historia, las prácticas, la enseñanza y las determinaciones del contexto sociocultural, en los cuales aparecen el tema del cuerpo, el juego, el movimiento, las prácticas corporales y los ideales y los fines de la enseñanza. Establece líneas de acción a las cuales debe corresponder la educación física contemporánea y que deben ser reconocidas en la construcción de la educación física como una disciplina de carácter práctico que se desarrolla como una pedagogía de la experiencia.

Palabras clave: Cultura intelectual, paradigmas de educación física, construcción teórica de la educación fisica.

\section{Abstract}

In the discussion about facts that transform the physical education in the Universidad Pedagógica Nacional and the national context, the topic of the relation between epistemology and physical education is very important. This article presents the concepts of the scientific epistemology from which it has developed the discussion of the physical education and its study subject, and expresses the option of considering the physical education as a practice. For doing this, it is based on the characterization made by Pierre Parlebas and the concept of episteme from Michel Foucault. This article proposes an investigation methodology from the fields of the history, practices, teaching and determinations in the sociocultural context, where appear the subject of the body, game, movement, physical exercises, ideals and the purposes of the teaching. It establishes guidelines to which the contemporary physical education must correspond and they must be acknowledged in the construction of physical education as a practical kind discipline that it is developed as an experience pedagogy.

Key words: Intellectual culture, physical education paradigms, theoretical construction of the physical education.

Fecha de recepción: 29 de octubre de 2005.

Fecha de aceptación: 8 de noviembre de 2005.

Este texto se elabora en el proceso de construcción de la fundamentación de la Especialización en Pedagogia y Didáctica de la . Educación Física y de la elaboración del proyecto sobre Epistemología e Historia y enseñanza de la Educación Física. Profesor de la Facultad de Educación Fisica de la Universidad Pedagógica Nacional. 


\section{Introducción}

Una interesante y en muchas ocasiones tensionante discusión se realiza en la Facultad de educación física de la Universidad Pedagógica Nacional con motivo de la creación del nuevo proyecto curricular de la licenciatura en educación física, que planteó una profunda renovación en el campo disciplinar de la educación física y en su correspondiente estructuración curricular (véase Fundamentación curricular PCLEF). A ello se suman la creación de programas de formación licenciados en deporte (véase Fundamentos Curriculares Licenciatura en Deporte) y en recreación (ver fundamentos curriculares Licenciatura en Recreación) y la actualización de las especializaciones para la acreditación previa ${ }^{1}$ que han generado toda clase de argumentos para justificar su pertinencia académica y social. Otros hechos de incidencia nacional manifiestan el movimiento de cambio que está dándose en la educación física colombiana: la expedición de los Lineamientos Curriculares de educación física por parte del Ministerio de Educación Nacional, que exponen un nuevo horizonte para la enseñanza de la educación física, la aprobación del Plan Nacional de educación física (véase Instituto Nacional del Deporte, Coldeportes...) que busca la articulación del conocimiento académico y la construcción social a través de nuevos escenarios de realización de la educación física para atender los diversos grupos de población, además de la escolar; y los debates que se producen en diferentes universidades en el contexto de cambios curriculares $^{2}$, la acreditación, la organización de la investigación y los programas de extensión social.

El surgimiento de una nueva realidad es evidente en el crecimiento de grupos de reflexión e investigación sobre diversos temas, muchos de ellos de carácter interinstitucional, estrechamente relacionados con la multiplicación de eventos académicos de las más variadas temáticas. En el ámbito social las prácticas, técnicas, empresas y programas relacionados con la actividad corporal son incontables. En el paisaje urbano ocupan un amplio espacio los gimnasios, los centros de condición física y de estética corporal y en los espacios públicos como parques, plazas y ciclovías se multiplican los participantes y la variedad de actividades.

En el campo de la educación se producen profundos cambios administrativos y pedagógicos y la enseñanza se cuestiona con el fin de encontrar alternativas correspondientes a nuevas realidades en comportamientos, gustos y significaciones que se expresan en lenguajes diferentes del de las regulaciones tradicionales. Temas estructurantes de la educación física, como el cuerpo, el movimiento, las prácticas corporales, el juego y las relaciones con la dimensión cognitiva y la cultura, se investigan desde diferentes visiones que corresponden a las nuevas sensibilidades e intereses que fluyen en el devenir contemporáneo.

El panorama muestra una dinámica académica, educativa y social que transforma la concepción de la educación física y su enseñanza y hace necesario la profundización en el proceso de investigación epistemológica y construcción disciplinar. Las preguntas tradicionales adquieren nuevos sentidos: ¿cómo se explica la educación física? ¿Cómo se relaciona la concepción de educación física con la enseñanza? ¿Qué contextos la determinan? ¿Qué horizontes se avisoran? ¿Cómo afrontar la construcción disciplinar?

El presente artículo expone algunos elementos que contribuyen a la reflexión sobre la educación física colombiana actual, su comprensión y sus tareas. Al respecto, se desarrollan tres ideas:

1. Una reflexión sobre la relación de la epistemología y la educación física, en la que se plantea un giro para dejar la obsesión de caracterizar la educación física como una ciencia y asumirla como una práctica que se apoya, entre otras, en la ciencia.

2. La investigación con miras a la construcción de la educación física como disciplina de orden práctico es inseparable de la enseñanza y se produce desde diferentes campos.

3. En el contexto contemporáneo se replantea la educación física a partir de los ejes de formación humana, construcción de vida, identidad cultural y pedagogía de la experiencia.

\section{Epistemología y educación física}

La epistemología posibilita mostrar los elementos e identificar los conceptos que guían la reflexión sobre la manera en que se construye el conocimiento en las ciencias y por correlación, en educación física, aspecto que aporta a la comprensión clara de la disciplina y de sus finalidades educativas y sociales. Al respecto, se reconoce una débil tradición en la formación epistemológica y teórica de los profesores de educación física de Colombia. Pese a ello el estudio de diferentes autores ${ }^{3}$ ha circulado con relativa amplitud en la comunidad académica, pero aislada de los efectos prácticos en la enseñanza y en la toma de decisiones políticas.

La tradición investigativa y docente, muestra la ubicación de la educación física en la frontera entre las cien- 
cias humanas y las ciencias naturales. Si se acepta que la educación física se apoya en conocimientos científicos, los criterios de la epistemología científica planteados por Piaget ilustran sobre sus condiciones y su uso. "El conocimiento constituye una cierta relación entre el sujeto (a) y un objeto (b) y las estructuras o formas (c) inherentes a todas las relaciones de conocimiento que unen sujetos (cualquiera que sea el nivel de estos conocimientos) con objetos (cualquiera que sea su variedad), y esto ya a partir de los objetos de la percepción" (Piaget. 1979). Esta relación se establece de acuerdo con unas condiciones constitutivas, que son las condiciones formales o experimentales de validez y las condiciones de hecho, relativas a los aportes del objeto y a los del sujeto en la estructuración de conocimientos.

El problema central de la epistemología consiste en establecer si el conocimiento se reduce a un mero registro realizado por el sujeto, de datos, ya bien organizados en forma independiente de él, en un mundo exterior (físico o ideal), o si el sujeto interviene de una manera activa en el conocimiento y la organización de los objetos (Ibíd., p. 16). Muy frecuentemente, el papel del sujeto escapa al análisis del conocimiento acabado, mientras que ese papel se impone con evidencia en el curso de los periodos de formación.

La epistemología estudia la constitución de los conocimientos válidos, (condiciones de acceso y condiciones de permanencia), a través de los cuales plantea su pregunta histórica: ¿cómo son posibles las ciencias? En la epistemología son importantes los métodos histórico-críticos y genéticos, de ahí que una segunda tarea de la epistemología sea el estudio del pasaje de los estados de menor conocimiento a los estados de conocimiento más avanzados (Ibid), que tienen su praxis en la investigación de la historia.

Desde otro enfoque, el profesor Francisco Lagardera Otero (1993, pp. 27-51), expone la teoría de Gastón Bachelard, autor clásico en la construcción de la epistemología. Bachelard entiende la epistemología como aplicabilidad racional de pensamientos, reflexiones y métodos que operan para vencer el límite del saber conocido. Distingue entre áreas epistemológicas y actos epistemológicos.

Las áreas epistemológicas corresponden a las parcelas del saber científico donde se ubica cada disciplina en función de su objeto de estudio y la generación de conocimientos específicos.

Los actos epistemológicos son las actitudes ante los he-chos que configuran una disciplina. El primero de ellos es la ruptura con la realidad inmediata, el segundo, la construcción del objeto científico, objeto construido que no tiene nada en común con las unidades delimitadas con la percepción ingenua; y el tercero, la contrastación entre el objeto construido y la manifestación de los hechos (Ibíd).

\section{¿Una ruptura?}

Vistos los anteriores enfoques, se pretende explorar otra posibilidad: en el trabajo epistemológico de la educación física es posible que se haya tomado el camino equivocado al asumir teorías que no corresponden a su naturaleza y que mantienen su producción en el plano de los discursos, sin lograr cambios efectivos en el plano de las prácticas de aprendizaje, enseñanza y socialización.

Sin desconocer la importancia de la epistemología científica, que guía hacia el esfuerzo, tal vez inútil, de justificar la educación física como una ciencia, debemos preguntarnos si el enfoque asumido es el que requerimos y si es necesaria una reorientación. En tal sentido debemos cambiar nuestra actitud frente a la idea de que lo único que hace importante a la educación física es considerarla como una ciencia. $\mathrm{Al}$ respecto, el punto de vista de Pierre Parlebas es muy claro:

\begin{abstract}
"Frente a la pregunta si la educación física es una ciencia, mi respuesta es categórica: La educación física no es ni será jamás una ciencia. La educación física es una práctica, una práctica pedagógica en el transcurso de la cual los profesores ejercen una influencia en los alumnos que les han sido confiados... Pero la educación física tiene relaciones con la ciencia y por ello quien la estudia debe tener una formación científica mínima... El profesor es un practicante pero puede estar esclarecido por conocimientos científicos. Mi respuesta es clara: la educación física no es una ciencia pero cada vez más debe ir apoyándose en los conocimientos científicos... Este último punto tiene dos caminos: o apoyarse en conocimientos exteriores de disciplinas yuxtapuestas, o en conocimientos propios" (Parlebas: 1997).
\end{abstract}

Con base en este planteamiento se expone una perspectiva de investigación que busca identificar elementos dispersos y reconocer los procesos históricos de surgimiento, evolución, constitución y ruptura de la educación física para poder tomar decisiones con criterio en el campo epistemológico y en su relación con la enseñanza. La investigación sobre construcción disciplinar en educación física debe reconocer una acumulación de conocimiento y experiencia y tener efectos prácticos hacia el campo de la enseñanza para superar esa especie de cientificismo estéril muy frecuente en muchos debates. 
A continuación se señalan cuatro campos de investigación considerados fundamentales en la construcción disciplinar de la educación física, al igual que los ejes alrededor de los cuales se dinamizan estos campos en la educación física contemporánea:
a. Investigar desde la historia
b. Investigar desde las prácticas
c. Investigar desde la enseñanza
d. Investigar desde los contextos social y cultural

En la constitución de estos campos intervienen como constantes interrelacionados el cuerpo, el juego, el movi-miento, las prácticas y técnicas corporales y los ideales y fines de acuerdo con el momento histórico y el contexto cultural.

\section{Investigar desde la historia}

La investigación de la historia de la educación física como soporte del proceso de construcción disciplinar requiere una transformación conceptual y metodológica que posibilite procesos de reconocimiento, comprensión y confrontación de los elementos que han constituido la educación física y estructurado su carácter en cada momento histórico y en los contextos de surgimiento y desarrollo. Diversas experiencias de investigación proporcionan las orientaciones sobre las cuales puede potenciarse el trabajo de conocimiento histórico.

Una opción metodológica para potenciar la investigación en la construcción disciplinar es la relación con la historia de la educación física, privilegiando la perspectiva arqueológica utilizada por Michel Foucault en la cual se define el concepto de episteme y las características del proceso de investigación.

Sobre el objeto de análisis entendido como episteme dice: "La episteme no es sinónimo de saber sino que es la expresión de un orden o, mejor dicho, del principio de un ordenamiento histórico de los saberes, principio anterior al ordenamiento del discurso efectuado por las ciencias e independiente de él (Machado: 1987). La arqueología abandona los criterios de verdad definidos por la ciencia y, aún más, por la actualidad de una ciencia. La arqueología realiza un doble desplazamiento de criterios: de la ciencia al saber y de la actualidad a la contemporaneidad. Sin neutralizar por completo la cuestión de la verdad, la arqueología no la toma como criterio de evaluación del pasado de la ciencia, sino que procura definirla en el seno del saber de la época estudiada a fin de establecer las condiciones de posibilidad de los discursos. Independientemente de la ciencia y de su actualidad, es la época misma la que define sus cri- terios epistémicos de verdad cuyo fundamento es el $a$ priori histórico"(Ibid).

Este enfoque ha sido utilizado por diferentes pensadores contemporáneos interesados en el tema del cuerpo y los resultados de sus investigaciones contribuyen a un cuestionamiento crítico de la educación física. Un ejemplo una historia desde este enfoque es Arqueología de la educación física, del investigador francés Jacques Gleyse.

Autores como Michael Bernard (1983), Michel Foucault (2002), André Rauch (1986), Jean Barreau y Jean Morne (1993), y en el ámbito local, Hernán Felipe Prieto (1996), Rubiela Arboleda (2004), Zandra Pedraza (1998) y Ximena Herrera, han realizado aportes muy destacados sobre el conocimiento múltiple, complejo y esquivo del cuerpo. En otros espacios el tema del cuerpo aparece como componente central en el nuevo currículo de la licenciatura en educación física de la Universidad Pedagógica Nacional y en los Lineamientos Curriculares del Ministerio de Educación Nacional.

Desde perspectivas diversas de investigación, el cuerpo como tema de la educación física ha sido objeto de reflexión de diversos autores, como Ommo Gruppe (1976) y José María Cajigal (1979), que han efectuado aportes para superar la visión orgánica y funcional de los enfoques fisiológicos y mecánicos.

El juego ha sido investigado por diferentes autores con influencia directa en el estudio de la educación fisica. Entre ellos se encuentran Johan Huizinga (Homo Ludens), Carl Diem (Historia de los deportes), Roger Callois (Los juegos y los hombres), Richard Mandel (Historia cultural del deporte). El juego se reconoce como componente fundante de la educación física y hace parte de su constitución histórica como contenido y como apoyo pedagógico y didáctico en distintos autores como AnneMarie Seybold (Principios didácticos de la educación física), Santiago Cocca (El hombre deportivo), Pierre Parlebas(Léxico para una ciencia...), Carlos Bolívar, Ximena Herrera.

El movimiento ha sido objeto de estudio de diferentes autores, entre los cuales se destaca por su visión totalizadora y sistemática Kurt Meinel y su obra clásica $D i$ dáctica del movimiento, elaborado a partir del enfoque del materialismo histórico.

Investigaciones como las sustentadas en los congresos colombiano y latinoamericano de historia de la educación física (2001 y 2005, respectivamente), presentan un abundante y rico material para la investigación epistemológica de la educación física desde una perspectiva histórica. 


\section{Investigar desde las prácticas}

El concepto de práctica es problemático, pero aquí lo entendemos como las acciones del cuerpo orientadas a fines, espontáneos o sistemáticos, que caracterizan la actividad de la educación física, a diferencia de otras prácticas, como las prácticas mentales de cálculo o las prácticas comunicativas, a través del lenguaje oral o la escritura. También corresponde a las prácticas el ambiente de su producción, los procedimientos y las tradiciones que conforman. Este planteamiento nos ilustra sobre una posibilidad metodológica para estudiar con rigor la extensa tradición de prácticas, saberes y técnicas ${ }^{4}$ que han conformado la educación física y reconocer las transformaciones en cada momento histórico, hasta la educación física que hoy conocemos y practicamos.

El desarrollo disciplinar de la educación física ha transformado el saber hacer práctico en un cuerpo de conocimientos, cuya apropiación requiere procesos de orden epistemológico y pedagógico muy definidos, que tienen su génesis en las prácticas de juego y movimiento, pero que se ha transformado y ha adquirido diversas significaciones.

Diferentes enfoques de estudio coinciden en reconocer como su especificidad, el carácter práctico de la educación física, cuyo propósito se centra en el saber hacer y en el saber cómo hacer. Su interés se basa en la obtención de propósitos a través de la acción, asociada a un razonamiento inteligente, el conocimiento del contexto, el carácter de la situación y las formas apropiadas de realización.

El concepto de práctica se produce en el desarrollo disciplinar y se basa en la comprensión de la acción y su relación con las características del sujeto y del contexto en el que se desenvuelve. La práctica como un saber hacer se complementa con la necesidad del saber cómo hacer y para qué hacer (Arnold: 1990). El saber cómo hacer está expresado en técnicas y formas de transmisión y apropiación, así como de las modificaciones que no desvirtúen su naturaleza. En el para qué hacer están implícitos fines y valores que a la vez que posibilitan una relación con la práctica aprendida son base para reorientar y transformar las propias prácticas o su sentido.

La especificidad del trabajo epistemológico de la educación física está en la comprensión de la naturaleza de las prácticas corporales en relación con una pedagogía, entendida como praxis, que orienta la formación humana.

\section{Investigar desde la enseñanza}

La enseñanza de la educación física es el problema actual que más preocupa a los profesores, a la comunidad educativa y a la sociedad. Los cuestionamientos son diversos: ¿qué estamos enseñando y para qué? ¿Cómo estamos enseñando? ¿A qué ideales responde la enseñanza? ¿Qué sentido tiene la educación física en la vida personal y en la cultura? (Fundamentos de la Especialización en Pedagogía y Didáctica de la educación física: 2005). Estas preguntas forman parte del objeto de reflexión epistemológica y señalan la naturaleza de la enseñanza de la educación física y sus características.

Una posible vía para abordar estas cuestiones es reconocer la enseñanza de la educación física como un proceso pedagógico en el que intervienen diferentes componentes interrelacionados, inseparables y determinantes unos de otros: el saber disciplinar, las prácticas de enseñanza y el contexto social y cultural en que se desenvuelve, cuya praxis educativa se expresa en la formación del sujeto y en la construcción de cultura y sociedad, aspectos que adquieren significado a través de la investigación epistemológica (Ibíd).

En la enseñanza de la educación física se ha conformado un conjunto de criterios y prácticas de carácter generalizado que podemos entender como tendencias que identifican la educación física real en nuestro medio(Grupo de Investi-gación, UPN: 2004). En la actividad educativa se ha impuesto una especie de eclecticismo que ha logrado cierta unidad de criterios en la estructuración de los currículos y en los métodos y medios de enseñanza. En general ${ }^{5}$, en la exploración de la educación básica primaria y en la secundaria realizada en diferentes instituciones pueden destacarse cuatro tendencias de enseñanza:

a. La "psicomotricista", cuya influencia se ejerce en los niveles de preescolar y básica primaria. Se basa en los aportes de Picq y Vayer y Jean Le Boulch, apoyada en la fuerte influencia de la psicología en la educación colombiana y en las tendencias pedagógicas centradas en el desarrollo del niño. Se combina en los grados cuarto y quinto con la tendencia "deportivista" a través de la inclusión de los predeportivos y minideportes.

b. La condición física y técnico-deportiva es predominante en la educación secundaria. Se denomina también "deportivista". Es influenciada por el proceso de deportivización, que tiene sus inicios en la década de 1960 y toma fuerza con la misión alemana y el convenio colombo alemán. Se fortalece con la tecnología educativa que se desarrolla en los programas del 
INEM. Se consolida en el contexto de deportivización de la sociedad, y con la extensión de las ciencias aplicadas al deporte, como la fisiología del ejercicio y la biomecánica. Su aplicación en la actualidad sigue siendo predominante, pero en su interior se han dado procesos complejos de transformación de prácticas y significaciones, como producto de cambios de la educación física y su visión pedagógica y cultural del deporte.

También se expresan otras tendencias:

c. Tendencia recreativa y lúdica. Influenciada por tendencias pedagógicas para hacer agradable y recreativa la actividad escolar, el avance en las ideas del derecho a la recreación, la extensión de los beneficios del juego en la formación y los procesos de construcción de alternativas para una educación física más creativa $\mathrm{y}$ dinámica.

En el imaginario estudiantil y social se entiende como una finalidad de la educación física: divertir, participar, integrar los grupos. También se relaciona con la salud.

d. Tendencia artística y de expresión corporal. Influenciada por el componente y la actividad artística y expresiva en la escuela. Promotora de la acción cultural en las instituciones y basada en la idea de rescatar y promover la identidad cultural a través de la danza, la ronda y el juego como manifestaciones de la cultura autóctona.

Al relacionar las tendencias o modelos epistemológicos con los enfoques o modelos de enseñanza, se encuentra la separación de las teorías del proceso efectivo y real de la enseñanza en diferentes formas: yuxtaposición, tensión o contradicción entre los componentes del proceso a través del cual el estudiante participa no sólo para aprender, sino también para formarse.

$\mathrm{Al}$ parecer en el campo académico se están elaborando y difundiendo teorías que no se aplican en la práctica sino en aspectos parciales, mientras que de la propia práctica no surgen las orientaciones que se requieren para afrontar las necesidades educativas. El problema se presenta tanto por la manera en que se construye la teoría, como en la forma de realizar la enseñanza, y se concluye en la cuestión del para qué la teoría si no parte de la realidad y ejerce en ella su poder transformador, a través de las prácticas que deben corresponderle.

Visto el panorama en el campo epistemológico y de la enseñanza, la reflexión se orienta a investigar y cons- truir las alternativas que integren estos dos campos inseparables del proceso educativo ${ }^{\top}$.

\section{Investigar desde los contextos social y cultural. Distanciamiento crítico}

Como área educativa fundamental y como práctica cultural, la educación física se encuentra afectada por diversos aspectos que la ponen en una situación de caminos contrapuestos. Mientras que en el plano social se acrecienta el interés por las prácticas relacionadas con la actividad física y con el cuerpo debido a la influencia de nuevos agentes educativos, como los medios masivos de comunicación, la publicidad y la información, en el nivel académico vive intensas discusiones y rompimientos para encontrar una respuesta efectiva a estas condiciones y exigencias sociales.

En el centro del cambio que vive la educación física se confrontan dos paradigmas: el hegemónico y el de la diversidad y la resistencia.

El paradigma hegemónico corresponde al actual modelo trasnacional de cultura y sociedad de mercado, individualización, competencia, eficiencia y calidad. Produce un modelo instrumental de la educación física que exige respuesta inmediata y rendimiento. Una representación de este paradigma en la enseñanza se expresa en la educación física como asignatura centrada en los ejercicios organizados de manera cuantitativa y competitiva y mecanicistas en el aprendizaje de la técnica. Es el ejercicio con finalidades puntuales y de rendimientos estandarizados a través de una evaluación que exige resultados precisos, reconocidos y rentables. Su fundamento conceptual coincide con los modelos de cuerpo estereotipado y consumista, y con la adquisición de bienes de consumo representados en vestuario, implementos y asistencia a determinados espacios que son símbolo de prestigio y relación social.

El Paradigma de la diversidad es el modelo que resiste el modelo dominante, que rescata la diversidad, que explora y busca centrarse en el ser humano, que recoge las experiencias emancipatorias y forma para la construcción de una mentalidad y actitud autónomas. Implica resolver el problema de si el reconocimiento de lo diverso es tan amplio y de cómo se encuentran los hilos conductores comunes que orienten y unan en la diversidad. Se representa en una educación física contextualizada en lo social y lo cultural, con una concepción de estudiante participativo, deliberante y democrático, amplia y flexible en sus contenidos y procesos metodológicos, exploradora de opciones e investigadora de la cultura y la idiosincrasia regional y local. 
La cuestión es si la educación física debe responder al modelo social dominante de carácter mercantil o tiene la potencialidad pedagógica suficiente para proponer alternativas diferentes de las de esta naturaleza.

\section{Líneas de acción en el momento actual}

El ser humano no sólo se mueve para sobrevivir como especie, sino también para resolver problemas y producir cultura desde su experiencia corporal y lúdica. Está predispuesto a los aprendizajes que le permitan una mejor calidad de vida $\mathrm{y}$ al desenvolvimiento en diferentes escenarios que le posibilitan construir diferentes estilos de vida y organización social, innumerables expresiones lúdicas, técnicas y usos del cuerpo, de acuerdo con las exigencias del modo de vida, el desarrollo productivo y los hábitos culturales para transformar la realidad e impulsar su propio desarrollo.

El horizonte planteado enmarca un contexto y unas condiciones para el trabajo epistemológico. Ello conduce a definir la educación física como disciplina académica, con tareas en el desarrollo del conocimiento y responsabilidades educativas y sociales, a partir de un fundamento que le da identidad: las prácticas corporales y su sentido. Se trata de reivindicar estas prácticas como el elemento o estructura de formación del hombre en todas sus dimensiones. Desde esta perspectiva la educación física como disciplina académica estudia todos los fenómenos que tienen que ver con el objetivo formativo del ser humano en sus diferentes dimensiones, a partir de la corporalidad, y con la manera en que las prácticas corporales se organizan en objetos de enseñanza, para su apropiación por parte de los miembros de la sociedad.

Transformar los procesos de enseñanza exige la relación entre el contexto cultural y el proceso de construcción disciplinar, desde la perpectiva de un paradigma crítico que integre dos componentes que aparecen separados: epistemología y enseñanza. El factor integrador está determinado por los conceptos de formación del ser humano y de cultura y la manera en que influyen en el desarrollo del conocimiento y la sociedad, cuestión que produce sentido, contenido y significación a la educación física ${ }^{8}$.

En el sentido planteado, el desarrollo de la educación física actual tiene como lineas de trabajo: atender al ser humano en sus necesidades de construcción de vida, fortalecer los principios de identidad cultural en el reconocimiento de la diversidad, formar las habilidades y competencias para la acción.
Atender el tipo de ser humano y las nuevas necesidades para vivir en el mundo actual

La enseñanza de la educación física debe atender el ser humano y su desarrollo pleno. Es una función no instrumental de la educación física y una opción por una educación abierta y múltiple. Educar para la emancipación humana a partir de la formación de valores de solidaridad, de sensibilidad y de relación armónica con el medio ambiente. Promover el aprendizaje de actuación con los otros, basados en una ética del reconocimiento de sí en los otros, lo cual exige una actitud crítica ante la competencia, el individualismo y la exclusión.

La enseñanza debe llevar al reconocimiento de sí, opuesto al concepto de cliente y consumidor. El reconocimiento de sí, como humano y como especie, (Morin) es la conciencia y el placer de descubrirse y actuar desde una pedagogía del deseo (Mockus), que supere una actitud individualista, por una actitud de ser; de potencia $\mathrm{y}$ no de instrumento.

Fortalecer patrones culturales propios que son la base de las identidades y de las posibilidades de aporte a la cultura

En la construcción de cultura la enseñanza de la educación física debe asumir el reto del multiculturalismo, el respeto a lo diverso, para responder a la vez a los elementos de unidad nacional que están en los valores éti$\cos$, estéticos y espirituales. Hacer una educación física para el reconocimiento de la diferencia y del aprovechamiento del conflicto como elemento dinamizador, y posibilitadora de la apropiación y defensa de la cultura propia a través de la construcción de sentidos de vida enriquecidos por la historia de cada cultura.

Educar en la vinculación a los espacios y al tiempo en el sentido de situarse y sentirse partícipe de la historia en la creación y encuentro con los elementos de identidad. Reconocer en la diversidad la riqueza multiétnica y multicultural, la diferenciación geográfica y ambiental y la relación con todos los aspectos de la vida y la defensa del modo de ser y de vivir en comunidad.

Formar las competencias que enriquezcan la experiencia humana

Los criterios expuestos para la enseñanza se desarrollan a través de prácticas corporales que abran espacios a la experiencia corporal, la experiencia sensorial, la actitud estética y lúdica en el sentido de expresión, creación y emancipación, a partir de una pedagogía de la educación física de apertura a la experiencia humana, caracterizada por: 
La experiencia de participación y solidaridad

Propone la opción para actuar como ser social en el interés común, y la responsabilidad en la propia acción y su incidencia en el bienestar propio y de los demás. Es no quedarse quieto ante la necesidad de actuar. Sensibilidad-ética.

\section{La experiencia de los sentidos}

Conduce a rescatar el sí mismo a través del sentir, en sus diferentes fuentes de experiencia de los sentidos. Orientar una educación sensorial. Lo sensible es la puerta de entrada a lo cognitivo, el reconocimiento de lo lúdico y la experiencia estética.

\section{La experiencia creativa}

Promueve situaciones para asumir el reto del pensar, la dificultad de explorar en el grupo, la importancia de compartir en público, el rechazo a la masificación, el esfuerzo por la participación, el reconocimiento de lo sencillo. Sensibilidad-cognición.

\section{Aprender a pensar en la acción}

tiene como punto de apoyo el desarrollo que Gardner llama la inteligencia cinestésico-corporal. Aprender a hacer con inteligencia. No la práctica por la práctica en sí, sino en el mejor sentido de realizar bien la tarea. Sensibilidad-técnica.

El fundamento metodológico se basa en explorar y descubrir, a partir de la investigación de los procesos planteados: aprender a ser social, aprender a sentir, aprender a pensar en la acción y aprender a crear y explorar modalidades de construcción-estructuración de la experiencia sensorial y corporal.

\section{Bibliografía}

ARBOLEDA, Rubiela. (2004). El cuerpo en boca de los adolescentes, Medellín. Kinesis.

BARREAU, Jean y MORNNE, Jean. (1992). Epistemología y antropologia del deporte, Barcelona, Editorial Alianza.

. (1993). Antropología y Epistemología del deporte, Barcelona, Alianza Editorial.

BERNARD, Michael (1983). El cuerpo, Buenos Aires, Paidós.

CAJIGAL, José María (1980). Cultura intelectual y cultura física, Buenos Aires, Kapeluz. Kapeluz.

(1979). Cultura intelectual y cultura física, Buenos Aires,

CALLOIS, Roger. (1998). Los juegos y los hombres, México, Fondo de Cultura Económica.

CHINCHILLA G., Victor Jairo (2004). Nuevos paradigmas de la educación física, Villavicencio, XCCEF.

COCCA, Santiago. El hombre deportivo. Barcelona. Alianza Editorial.

CONGRESO COLOMBIANO Y LATINOAMERICANO DE HISTORIA DE LA EDUCACIÓN FÍSICA. (2001 y 2005) Documentos, Bogotá, Universidad Pedagógica Nacional.

DE ZUBIRÍA, Miguel (2005). Pedagogía conceptual. Bogotá. Fundación Internacional Alberto Merani.

DIEM, Carl. Historia de los deportes. Barcelona, Luis de coralt.

FOUCAULT, Michel (2002). Vigilar y Castigar. Historia de la sexualidad. Editorial Siglo XXI.

FUNDAMENTACIÓN CURRICULAR, Licenciatura en Recreación, Facultad de Educación Física (2005). Bogotá. Universidad Pedagógica Nacional.

FUNDAMENTACIÓN CURRICULAR DEL PCLEF. (1996). Facultad de educación física, Bogotá. Universidad Pedagógica Nacional.

FUNDAMENTOS CURRICULARES, Licenciatura en Deporte, Facultad de Educación Física (2005). Bogotá. Universidad Pedagógica Nacional.
FUNDAMENTOS DE LA ESPECIALIZACIÓN EN PEDAGOGÍA Y DIDÁCTICA DE LA EDUCACIÓN FÍSICA, (2005). Bogotá. Universidad Pedagógica Nacional. 1995.

GLEYSE, Jacques, Archéologie de l'éducation physique au XX $\mathrm{XX}^{\mathrm{em}}$ - siècle en France. Presses Universitaires de France.

GRUPO DE INVESTIGACIÓN, Universidad Pedagógica Nacional (2004). Paradigmas en la Enseñanza de la Educación Física, Proyecto en realización. Bogotá. Universidad Pedagógica Nacional.

GRUPPE, Ommo. (1970). Teoría pedagógica de la educación física, Madrid. INEF. drid, INEF.

(1976). Teoria antropológica de la educación fisica. Ma-

HUIZINGA, Johan, Homo Ludens.

INSTITUTO NACIONAL DEL DEPORTE, Coldeportes.(2003). Plan Nacional de Desarrollo de la educación física, Conocimiento y Construcción Social, Bogotá.

KIRK, David. Educación fisica y curriculo, Valencia. Universidad de Valencia.

LAGARTERA OTERO, Francisco (1993). Bases epistemológicas de la educación física escolar. Actas Simposio Internacional sobre Educación Física Escolar y Deporte de Alto Rendimiento, Palmas de la Gran Canaria.

LANGLADE, Alberto, REY, Nelly (1976). Teoría general de la gimnasia. Stadium, Buenos Aires.

LE BOULCH, Jean (1987). Hacia una ciencia del movimiento humano, Paidós. . (2003). Pedagogías del cuerpo en el siglo XXI, Barcelona, INDE.

LLINAS, Rodolfo (2004). El cerebro y el mito del yo, Bogotá, Norma.

MACHADO, Roberto (1987). Arqueología y epistemología, del libro Ciencia e saber; a trajetoria de arqueologia, de M. Foucault, Rio de Janerio, Graal, 2 edición. 
MANDEL, Richard. Historia cultural del deporte.

MARIO (1970). Memoria del VI Curso Internacional de educación física, Bogotá.

MEINEL, Kurt. (1978). Didáctica del movimiento, La Habana, Orbe.

Ministerio de Educación Nacional. (2000). Lineamientos Curriculares en educación física, Deporte y Recreación, Bogotá.

MOCKUS, Antanas, HERNÁNDEZ, Carlos, CHARUM, Clemencia. Las fronteras de la escuela.

MORALES, Leonel (1990). Construcción teórica de la educación fisica, Bogotá. ACPEF,

PARLEBAS, Pierre (1998). Léxico para una ciencia de la acción motriz. París. INSEP.

- Léxico para una ciencia d e la acción motriz; Las cuatro esquinas de los juegos.

. (1997). Problemas Teóricos y Crisis Actual en la Educación Física Conferencia realizada en el Centro de Alto Rendimiento Deportivo (CE.NA. R.D.), Buenos Aires. Secretaría de Deportes. PEDRAZ, Vicente. Epistemología d e la actividad física, Madrid, Gimnos.

\section{Notas}

1 Véase documentos de Fundamentación Curricular de las especializaciones en Pedagogía y Didáctica de la Educación Física, Pedagogia del Entrenamiento Deportivo, Administración de la Educación Física, el Deporte y la Recreación, Facultad de Educación Física, Universidad Pedagógica Nacional, Bogotá, 2005.

2 Véase Reformas Curriculares de Programas de Formación en Educación Física, de la Universidad de Antioquia y la Universidad del Cauca.

${ }^{3}$ El trabajo incluye una breve identificación de autores representativos de la reflexión epistemológica de la Educación Física.

4 Véase por ejemplo, Teoría general de la gimnasia, de Alberto Langlade y Nelly Rey de Langlade, Stadium.
PEDRAZA, Zandra (1998). En cuerpo y alma. Imágenes del cuerpo y la felicidad, Bogotá. Universidad de los Andes.

PETER, Arnold (1990). Educación, movimiento y curriculum, Morata.

PIAGET, Jean (1979). Naturaleza y métodos de la epistemología, Buenos Aires. Paidós.

PRIETO, Hernán Felipe (1996). Seminario del Cuerpo, Especialización Pedagogía y Didáctica de la educación física, Universidad Pedagógica Nacional.

RAUCH, André (1986). El cuerpo en la educación física, Buenos Aires. Kapeluz.

RODRÍGUEZ LÓPEZ, Juan (1995). Deporte y ciencia, teoría de la actividad fisica, Barcelona, INDE.

SEYBOLD, Annemarie (1978). Principios didácticos de la educación física, Buenso Aires, Kapeluz.

Sobre el concepto de sentido, contenido y significación ver: Dallo, Alberto, Lopez.

TRIGO, Eugenia y colaboradores (1999). Creatividad y motricidad, Barcelona, INDE.

5 Documentos de preparación de lineamientos curriculares del Ministerio de Educación. Véase también las conclusiones de los 12 encuentros regionales de Educación Física en Cundinamarca, realizado en el año 2003. Véase avances del Proyecto de Investigación Relación Teoría y Práctica en la Enseñanza de la Educación Física. Facultad de Educación Física, UPN.

6 Los términos "psicomotricista", "deportivista", "biologista", no corresponden al autor de este artículo. Son expresiones muy utilizadas en el medio.

7 Esta perspectiva es asumida como objeto de estudio de la Especialización en Pedagogia y Didáctica de la Educación Física.

8 Sobre el concepto de sentido, contenido y significación, véase Dallo y otros. 